\title{
Editorial
}

\section{The Society for Neuroscience 2009 Meeting Report, Part 2}

\author{
Amber Dance, Esther Landhuis and Gabrielle Strobel \\ Alzheimer Research Forum
}

\section{TAU AND $\alpha$-SYNUCLEIN OLIGOMERS FOLLOW A $\beta$ FOOTSTEPS}

In Alzheimer's disease research, focus has recently shifted away from large plaques and toward small oligomers of amyloid as a potential cause of disease [1, 2]. At the Society for Neuroscience annual meeting held 17-21 October 2009 in Chicago, Illinois, dozens of presentations dealt with $\mathrm{A} \beta$ oligomers, but what stood out more as a budding trend were signs that research in the tau and $\alpha$-synuclein fields may be going down the same road. Several presentations covered emerging tools to study oligomers of these two proteins, and they drew wide notice among attendees.

\section{Untangling tau}

Oligomers form shapes distinct from either monomer or fibrillar aggregated forms. Accordingly, researchers can make antibodies specific for particular conformations. Rakez Kayed described how his group at the University of Texas Medical Branch in Galveston pursued a similar approach to generate first tau oligomers, then antibodies against them, that had borne fruit while he was a postdoc in Charlie Glabe's laboratory at the University of California, Irvine [3]. In his Galveston laboratory, Kayed seeded recombinant full-length human tau with $\mathrm{A} \beta$ oligomers or with $\alpha$-synuclein oligomers. "I think this way of making oligomers is relevant to what happens in vivo," Kayed told ARF. The scientists used the resulting tau oligomers as an antigen and produced a series of anti-tau antibodies that they claim recognize trimers and higher-order oligomers, but not monomers or fibrils.

The laboratory gave several presentations in Chicago. In a slide talk, Kayed focused on tau oligomers with a distinct ring-like shape. The story starts with work on amyloid- $\beta$, which can form distinct pore-like structures called annular protofibrils. As postdoc, Kayed had raised an antibody, dubbed Officer, specific for these shapes. He and Glabe found that Officer also nabbed other pore-forming proteins, suggesting it is specific not for amyloid- $\beta$, but for the $\beta$-barrel structural motif of the pore [4]. In Chicago, Kayed described how when he used Officer to detect amyloid- $\beta$ annular protofibrils in brain tissue from people who had had Alzheimer disease, he noticed that some of the labeled annular structures contained no amyloid- $\beta$ and looked suspiciously like structures commonly formed by tau. Sure enough, double-immunostaining with tau antibodies and Officer suggested that the AD brain contains tau, as well as amyloid- $\beta$, annular protofibrils. The scientists found similar tau annular protofibrils in tissue from people who had dementia with Lewy bodies (DLB) and a tauopathy called progressive supranuclear palsy. The researchers apparently were also able to make Officerreactive tau oligomers in vitro; on a Western blot, these tau annular protofibrils ran as a smear with a molecular weight more than $50 \mathrm{kDa}$, Kayed reported.

Kayed suggested that tau annular protofibrils might form pores in the cell membrane, allowing ions to pass through and disrupting cellular homeostasis. This might be true especially in astrocytes and oligodendrocytes, which contain more annular tau structures than 
do neurons, Kayed said. Because the annular protofibrils are ubiquitinated, they might also impede the cell's protein destruction pathway, he added, allowing other misfolded proteins to accumulate.

On a poster, Cristian Lasagna-Reeves, a graduate student in Kayed's laboratory, presented a similar approach with a different antibody that detects tau oligomers. Lasagna-Reeves noted that the presence of neurofibrillary tangles correlates less tightly with symptoms of AD than does the presence of pre-fibrillar tau. "We think [tau oligomers] are the real toxic species in Alzheimer disease," Lasagna-Reeves said, adding that his work now gives researchers a method to detect those species.

Called T2286, his new antibody detects 109-kDa spherical oligomers, but not monomers or fibrils, nor other kinds of amyloid-forming protein. Using brain tissue extracts, Lasagna-Reeves reported that frontal cortex from people who had had AD showed immunoreactivity with T2286; control samples did not. Using CSF from some 20 people, he reported an increase in T2286 immunoreactivity in people with AD relative to normal controls, suggesting spherical tau could eventually become a biomarker for the disease as well as a potential therapeutic target. This early CSF data was not presented alongside established CSF tau assays, for example, the INNOTEST htau or phosphotau ELISAs widely used in clinical AD research across the world, and no absolute concentrations of the tau oligomers in CSF were given on the poster. Therefore, a comparison of the proposed tau oligomers to prior data on CSF total tau or phospho-tau concentrations in $\mathrm{AD}$ and controls was not possible from this initial study. In the next few months, the Texas group is planning to measure tau oligomers in a large number of CSF and serum samples, Kayed noted.

In a conversation with ARF, Kayed said his laboratory did do a comparison both in CSF and brain extracts, that is, with various research antibodies against phosphorylated forms of tau. It showed that less than 30 percent of the tau oligomers his new antibody detected were phosphorylated. "This surprised us. We had expected it would be more," Kayed said. Whether phosphorylated tau might act as a seed, or whether phosphorylation might not occur until after filaments have formed, remains debatable, Kayed noted. Beyond AD brain, the T2286 antibody detected tau oligomers in brain extracts of dementia with Lewy bodies, supranuclear palsy, and Parkinson disease. In addition, T2286 picked up tau oligomers in the P301L and rTg4510 tau mouse models, as well as an $\alpha$-synuclein mouse model, the Tg2576, and an A $\beta$ PP/PS1 line, Kayed said.
In the $\mathrm{rTg} 4510$ regulatable tau mouse, the presence of oligomers correlated with a behavioral phenotype Karen Ashe's group at the University of Minnesota, Minneapolis, had demonstrated previously, whereby memory function recovers when the tau transgene gets switched off even as neurofibrillary tangles stay in place $[5,6]$. "Our new contribution to that is showing specifically with our antibody that the oligomers are gone at this point of behavior improvement," Kayed said. He added that other unpublished experiments indicate that these oligomers are toxic to cultured cells, and that scientists at University of Texas Medical Branch plan to begin humanizing this antibody toward a tau immunotherapy [7] and to elucidate the epitope recognized by this antibody.

Overall, other scientists were intrigued by this talk and poster. However, they cautioned that the prospect of a single antibody that specifically recognizes neurotoxic tau oligomers in Western blots, ELISA, on tissue sections, in CSF, and that can serve as a start for tau oligomer immunotherapy sounds almost too good to be true and will need careful experimental substantiation.

In another poster, Kristina Patterson, a graduate student in the laboratory of Skip Binder at the Northwestern University School of Medicine in Chicago, presented her work on tau oligomers. Patterson used chemical cross-linking to stabilize tau oligomers in vitro. Typical cross-linkers rely on tau's two cysteines to hook peptides together, but that limits the linkable conformations to those with two cysteines in proximity. "We decided we wanted to give it more choices," Patterson said. She used a benzophenone cross-linker that binds cysteine with one end, but any carbon-hydrogen bond with the other. The non-specific end is activated by ultraviolet light. In other words, Patterson allowed the cross-linker to bind the cysteines and then gave the tau molecules the opportunity to oligomerize before hitting them with UV light to stabilize whatever conformation they happened to be in.

Patterson rather expected to see a ladder in her gels, with step-like increases for each additional tau in the oligomer. Instead, she saw mostly a species that ran at $180 \mathrm{kDa}$. The researchers also discovered a $180-\mathrm{kDa}$ tau oligomer in brain homogenates from four people who had $\mathrm{AD}$; the oligomer was not present in control samples. The $180 \mathrm{kDa}$ corresponds to a trimer, but Patterson noted that other tau combinations could run at that weight depending on their structure. Patterson is currently characterizing the exact makeup of the oligomers, but has not yet performed size exclusion chromatography to show definitively that the bands 
from the $\mathrm{AD}$ sample are oligomers. In general, the study of tau oligomers is in its infancy, Patterson said, opening up many new possibilities for research. "These results overall are similar to what we see," Kayed told $\mathrm{ARF}$ at the conference.

\section{Un-aggregating $\alpha$-synuclein}

Test-tube cross-linkers are also contributing to $\alpha$ synuclein research. Martin Ingelsson presented a talk on work he and Joakim Bergström are doing at Uppsala University in Sweden. They used reactive aldehydes; these are compounds formed in the body under conditions of oxidative stress, a likely contributor to $\alpha$-synucleinopathies. The researchers compared $\alpha$-synuclein oligomers cross-linked by either 4hydroxy-2-nonenal (HNE) or 4-oxo-2-nonenal (ONE). Both of these reactive aldehydes converted $\alpha$-synuclein monomers into stable, $\beta$-sheet rich structures of approximately $2,000 \mathrm{kDa}$, which Ingelsson estimates contain 40 to 50 monomers. However, atomic force microscopy revealed that the ONE-induced $\alpha$-synuclein oligomers were amorphous and variable in size, while HNE-induced oligomers formed distinct donut-shaped structures that are similar to the annular protofibrils described for amyloid- $\beta$ and tau. None of these oligomers aggregated further into fibrils such as seen in Lewy bodies. This supports a suggestion made previously by Glabe, Paul Muchowski, and others that oligomers can form in their own side pathways that dead-end with the oligomeric state and do not continue on to large fibrils.

Ingelsson further reported that both types of oligomer were taken up by cultured cells, where they proved cytotoxic. "This is just another example of commonalities" in diseases based on amyloid-forming proteins, Ingelsson said. Based on this research, the Uppsala group is hoping to develop a future $\alpha$-synuclein immunotherapy along the lines of an anti-amyloid- $\beta$ oligomer antibody originally developed in their laboratory, which is in late preclinical development at present.

Last but not least, Karin Danzer, a postdoctoral researcher in the laboratory of Pam McLean at Massachusetts General Hospital in Charlestown, presented new data on how she detected $\alpha$-synuclein oligomers secreted from living cells. The poster generated a crowd and persistent buzz among scientists. "This demonstration of $\alpha$-synuclein oligomer secretion is very attractive, because it implies these species will be more accessible to therapeutic removal than previously thought," commented Dennis Selkoe of Brigham and Women's Hospital in Boston. Danzer characterized the trans- mission of oligomers through cell media. The group combined culturing neuroglioma cells in a microfluidic chamber with an assay they developed to determine if extracellular $\alpha$-synuclein was monomeric or oligomeric. Danzer generated two $\alpha$-synuclein fusions: one linking the protein to the amino terminus of luciferase, and one linking it to the carboxyl terminus. Alone, the fusion proteins produced no luminescence. Together in an oligomer, the luciferase domains worked together to release light, which Danzer measured to quantify oligomerization.

The researchers found that $\alpha$-synuclein oligomers, ranging in size from $14 \mathrm{kDa}$ to $40,000 \mathrm{kDa}$, were secreted by neuroglioma cells. In previous work, the same group had shown that the chaperone Hsp70 reduced $\alpha$-synuclein aggregation [8]. In the new assay, they found that co-transfecting Hsp70 reduced the secreted oligomer signal by 24 -fold, without decreasing the amount of $\alpha$-synuclein present. The data suggest that Hsp70 blocks oligomerization without destroying the protein.

\section{NICOTINIC AChRs: $\alpha 4 \beta 2$ IFFY FOR AD, MORE PROMISE WITH $\alpha 7$ ?}

Days before the masses swarmed to Chicago for the Society for Neuroscience annual meeting, a more intimate assembly of 287 exchanged the latest buzz on nicotinic acetylcholine receptors (AChR) in a satellite symposium held 14-16 October in the northwest suburb of Lincolnshire. The 33 talks and 81 posters on the agenda ran the gamut from basic biology to drug development, and described therapeutic applications in smoking, addiction, and pain, as well as cognition. This story features clinical updates for a number of nicotinic AChR compounds in development for cognitive indications.

Therapeutic approaches targeting nicotinic AChRs for cognition have focused primarily on two subtypes - high-affinity $\alpha 4 \beta 2$ and low-affinity $\alpha 7$ receptors. These neuronal membrane proteins adorn the cortex, hippocampus, and thalamus - the brain's prime centers for learning and memory. Positron emission tomography (PET) studies show that expression of the $\alpha 4 \beta 2$ receptor drops in affected brain areas of patients with mild cognitive impairment (MCI) and Alzheimer's disease (AD), and $\alpha 4 \beta 2$ availability seems to correlate with severity of cognitive impairment [9]. The situation with $\alpha 7 \mathrm{nAChRs}$ has been more controversial, though in the clinic compounds targeting these receptors seem to have the upper hand on $\alpha 4 \beta 2$ agents, which have fared poorly thus far in trials for $\mathrm{AD}$ and schizophrenia. 


\section{A $\beta 4 \beta 2$ - slow going}

In a talk by Ed Johnson of AstraZeneca in Wilmington, Delaware, the clinical outlook for the selective $\alpha 4 \beta 2$ agonist AZD3480 (TC-1734) looked cloudy at best. Discovered by partner company Targacept in Winston-Salem, North Carolina, this compound appeared promising in rodent models of episodic memory, working memory, and spatial memory, and has been safe and well tolerated in numerous clinical studies. However, efficacy signals have yet to appear reliably. Earlier this year, the company reported, and Johnson reiterated in Lincolnshire, that a three-month Phase IIb trial of the $\alpha 4 \beta 2$ agonist in mild to moderate AD patients was inconclusive based on primary outcome. Not only did the treatment group fail to show meaningful change in ADAS-Cog scores at week 12 compared to baseline, but even participants taking donepezil (i.e., symptomatic drugs among the AD standard of care) did not show an uptick at 12 weeks, relative to those on placebo. A company news release attributes these results in part to unexpected improvement in the placebo group, a much-debated feature of recent AD trials. On safety and tolerability, the $\alpha 4 \beta 2$ agonist was similar to placebo and had fewer gastrointestinal-related adverse events than did donepezil. In a separate Phase IIb trial, AZD3480 did not improve cognitive deficits in schizophrenia patients who were taking an antipsychotic drug, Johnson said.

A ray of hope appeared in a more recent Phase II trial of adults with attention deficit hyperactivity disorder (ADHD). In that study, people on the higher dose $(50 \mathrm{mg})$ showed improvement on the primary outcome measure (total symptom score on the Conners Adult ADHD Rating Scale-Investigator Rating), and the compound was not associated with any serious adverse events. AstraZeneca plans to continue development of AZD3480 for ADHD, and is recruiting for a Phase I study of another selective $\alpha 4 \beta 2$ agonist, AZD1446 (TC-6683), for AD.

Jeffrey Baker of Abbott Laboratories in Abbott Park, Illinois, updated the audience on ABT-089, an $\alpha 4 \beta 2$ partial agonist that improves working memory and selective attention in monkeys. The compound has also been safe and well tolerated in six Phase I studies totaling 198 healthy adults. However, earlier this year the company terminated a Phase II trial testing ABT-089 as an adjunct therapy in mild to moderate AD patients already taking cholinesterase inhibitors. This 12-week study enrolled nearly 400 people at 39 US sites. It used a new trial design called response-adaptive randomiza- tion, whereby primary efficacy data are evaluated every two weeks in a sponsor-blinded fashion. This allows participants to be shifted into the most informative treatment arms as the study proceeds. Another benefit of the adaptive trial design is the pre-specification of futility criteria that warrant termination before the study proceeds to its bitter end. In other words, things fail faster, allowing sponsors to redirect the saved time and money toward other projects. Indeed, Abbott did terminate the AD trial of its $\alpha 4 \beta 2$ compound after an interim analysis revealed that none of the six treatment arms was separating from placebo on the study's primary outcome measure, the ADAS-Cog. Like the AstraZeneca $\alpha 4 \beta 2$ compound, ABT-089 looked promising in a four-week Phase II study of adults with AD$\mathrm{HD}$; it improved some ADHD symptoms, relative to placebo, but that trial was not powered to assess cognition. Abbott has since decided to discontinue ABT-089 studies in ADHD, a company representative confirmed by e-mail.

\section{Lucky seven?}

In an overview of nAChRs in drug discovery, Steve Arneric of Eli Lilly and Company in Indianapolis, Indiana, speculated whether AD drug developers have been barking up the wrong tree in their attempts to target $\alpha 4 \beta 2$ receptors. He noted several lines of research hinting that $\alpha 7 \mathrm{nAChRs}$ are the way to go. In a study published several months ago, treatment with an $\alpha 7$ agonist (S 24795) brought functional recovery to cortical synaptosomes from AD patients by disrupting interactions between $\mathrm{A} \beta$ peptides and $\alpha 7$ receptors [10]. Earlier this year, scientists reported that lack of $\alpha 7$ protected AD mice from $\mathrm{A} \beta$-induced synaptic loss, restored long-term potentiation, and improved cognition [11]. In addition, researchers recently reported a new $\alpha 7$ nAChR subtype, the $\alpha 7 \beta 2$ receptor, which may not only outnumber homomeric $\alpha 7$ receptors in the basal forebrain, but also seems more sensitive to blockage by $\mathrm{A} \beta$ oligomers [12]. Finally, a recent review proposes that $\alpha 7$ receptors may link a number of apparently disparate mechanisms thought to underlie AD [13].

Judging by talks and posters at the nAChR symposium, $\alpha 7$ compounds are outpacing $\alpha 4 \beta 2$ compounds in the clinic, and the field's hot new pursuit - positive allosteric modulators (PAMs) - could be poised to dethrone the more tried-and-true agonists. But it's still early days with the PAMs, which differ from agonists in that they target sites away from the substrate binding action. Several $\alpha 7$ PAMs look decent in preclinical 
studies, but only one has gotten the go-ahead for Phase I trials. At the meeting, many scientists were unable, or unwilling, to confidently say whether modulators would ultimately beat out agonists in the clinic.

In the meantime, several $\alpha 7$ agonists have undergone early Phase II studies and, more importantly, survived them to stay in the running for future trials. Tanya Wallace of Roche in Palo Alto, California, briefed attendees with clinical data on the company's $\alpha 7$ partial agonist R3487 (MEM3454), developed in collaboration with Memory Pharmaceuticals, which Roche acquired last year. In a Phase IIa proof-of-concept monotherapy study, mild to moderate AD patients receiving the lowest dose $(5 \mathrm{mg}$ ) showed improvement in accuracy and speed of memory in the study's primary endpoint, the CDR test battery. "As we increased the dose (to 15 or $50 \mathrm{mg}$ ), the pro-cognitive improvement was reduced," Wallace said, noting this trend had also appeared in preclinical analyses. She said the dosing effects could stem from one of the $\alpha 7$ nAChR's key features - its tendency to rapidly desensitize upon prolonged agonist exposure. Earlier this spring, the company began recruitment for a Phase IIb study that aims to enroll 420 mild to moderate AD patients at 62 worldwide sites. This is a six-month trial testing a lower dose range (1, 5 , or $15 \mathrm{mg}$ ) as an add-on to donepezil treatment, with ADAS-Cog as the primary endpoint, Wallace said. A poster by Wallace and other researchers at Roche and Memory Pharmaceuticals showed that the compound improves attention and working memory in non-human primates with properties in line with clinical data.

Gerhard Koenig spoke of another $\alpha 7$ agonist with promising Phase II signals - EnVivo Pharmaceuticals' EVP-6124. This compound is "very selective" and has "truly superior brain penetration," which would mean limited systemic exposure, Koenig noted in an e-mail to ARF. "We believe that could be a key unique feature of our compound," he wrote. In a poster presentation at this year's International Conference on Alzheimer's Disease (ICAD) in Vienna, Koenig's team reported cognitive benefit (measured as improvement on some parts of the CogState or NTB batteries) in mild to moderate AD patients who received EVP-6124 for four weeks as an adjunct therapy with acetylcholinesterase (AChE) inhibitors. That trial was small (48 patients). A longer Phase IIb AD study involving more than 200 participants is set to begin in the first half of 2010, Koenig said. He noted in an e-mail that primary endpoints for this trial are still being intensely discussed and cannot at this point be disclosed publicly. EnVivo is also wrapping up data analysis of a Phase Ib/IIa monotherapy trial of its $\alpha 7$ agonist in AD patients who did not take AChE inhibitors, Koenig said. Meanwhile, the Watertown, Massachusetts-based biopharmaceutical company plans to move ahead with a three-month Phase IIb trial of its $\alpha 7$ agonist in schizophrenia patients by late 2009 , based on promising biomarker Phase Ib data. The schizophrenia trial will test EnVivo's compound on top of existing antipsychotics, using parts of the CogState and NTB batteries as primary endpoints and functional measures from a new interview-based assessment of cognition, the Schizophrenia Cognition Rating Scale (SCoRS), as secondary endpoints.

Targacept, Inc. of Winston-Salem, North Carolina, also has an $\alpha 7$ agonist in the running - at this point for treatment of cognitive dysfunction in schizophrenia. The compound (TC-5619) is a full agonist that is selective for $\alpha 7$ and, unlike many other $\alpha 7$ compounds, does not hit 5-HT serotonin receptors. In rat models of social interaction and novel object recognition, the compound worked best at lower doses, and its pharmacokinetics and safety profiles have looked good in Phase I studies. The take-home message from Pat Lippiello's talk at the nAChR symposium was that $\alpha 7$ compounds have potential not only to help with cognitive dysfunction, but also with other symptoms such as withdrawal and flattened affect in schizophrenia. The company plans to begin recruitment for a Phase II proof-of-concept trial of its $\alpha 7$ compound in schizophrenia later this year, and will consider clinical development of the agonist for AD and ADHD going forward, Lippiello said.

Partnering with Siena Biotech in Siena, Italy, Wyeth also has an $\alpha 7$ agonist (WYE-103914/SEN34625) in the works. This compound is a full agonist that steers clear of other nAChR subtypes and 5-HT receptors. It uses a flex-bridge chemical platform thus far unique to $\alpha 7$ compounds in in vitro assays. This agonist seems to hit a different pathway; it increases glutamate transmission, whereas other $\alpha 7$ agonists have only elicited increases in dopamine and acetylcholine signaling. Whether these features are advantageous or disadvantageous remains to be seen, said Wyeth chemist Simon Haydar, a coauthor on two posters showing preclinical data on the compound. WYE-103914/SEN34625 has shown pro-cognitive and potential neuroprotective activities in rodent studies. In addition, the agonist seems able to improve cognition without affecting the activity of an antipsychotic drug offered in combination. This property would be critical for future drugs treating cognitive dysfunction associated with schizophrenia. 


\section{NICOTINIC AChRs - MECHANISTIC BASIS FOR NEW DRUG DISCOVERY?}

All too often, basic science findings that initially seem promising never translate into viable pharmaceutical approaches, or when they do, fail miserably in clinical trials. Amid such tales of disappointment, it's refreshing to hear about studies showing clear convergence between biology, drug action, and behavior, all in the space of a few seconds, no less. At a satellite symposium on therapeutic approaches targeting nicotinic acetylcholine receptors (nAChRs), which took place days before the Society of Neuroscience's annual meeting in Chicago last month, Martin Sarter of the University of Michigan, Ann Arbor, had such a story to tell. His laboratory developed a way to record neurotransmitter release at sub-second resolution in freely moving animals. Using this technology, the researchers identified an electrophysiological readout for key attention tasks in rodents. Better yet, treatment with nicotine or nAChR agonists can tweak this readout and, correspondingly, affect task performance. "The neuroscience and the pharmacology come together," Sarter told ARF. "That's something you don't see very often in this business." The findings could have implications for screening new nAChR-targeting drugs.

Noting its role in regulating sleep/wake cycles and other types of arousal setting, scientists had presumed that the cholinergic system operates on the level of minutes, even tens of minutes. But once Sarter and colleagues pioneered their technology for listening to neurotransmitter release in real time, they made a surprising discovery. By recording acetylcholine release from presynaptic terminals in the prefrontal cortex of rats engaged in a cue detection task, the researchers saw characteristic "transients" - bursts of cholinergic activity - that mediated task performance on a timescale not of minutes, but of seconds [14]. "That's really, really short," Sarter said. "It was a whole new story."

The rats in these studies wore surgically implanted, choline-sensitive microelectrodes, and were trained to perform a sustained attention task involving repeated rounds of pressing one lever if a cue light went on and pressing another lever if the light remained off. The researchers measured the accuracy of cue detection as well as response speed and other parameters. When they treated the rats with mecamylamine to block nACh receptors, the cue detection rate dropped considerably; this indicates that the nAChR system is required for this attention task and for generating the underlying electrophysiological signature, that is, the rapid tran- sients. Quicker detection rates were found to be associated with larger amplitudes and faster decay rates of cholinergic transients, Sarter said.

For the next phase of analysis, the researchers made the attention task harder by introducing a distractor for example, house lights flashing on and off. This lowered the baseline task performance and made the system more useful for determining how well various treatments influence the transients and, in turn, improve behavior. With the lower baseline, behavior was measured in terms of how long it took the rats to recover normal (i.e., distractor-free) levels of performance. The researchers found that nicotine helped the rats detect cues faster; importantly, this corresponded with larger amplitudes on their transients compared to vehicle treatment. Treatment with Abbott's $\alpha 4 \beta 2$ agonist ABT-089 improved task performance even more, and further sharpened the transients - that is, gave them higher amplitude and faster decay rate. However, treatment with an $\alpha 7$ agonist (ABT-107) did not help attention, and increased the duration of acetylcholine release. The readout for this was cholinergic transients with a longer "tail."

Abbott has discontinued development of ABT-089 for $\mathrm{AD}$ and $\mathrm{ADHD}$, in part because the $\alpha 4 \beta 2$ agonist failed to show efficacy in a recent Phase II AD trial. Sarter does not necessarily find these clinical disappointments inconsistent with his rat studies, where the compound has shown promise. "It is difficult to see how such a compound would work in $\mathrm{AD}$, given that the prefrontal 'cue detection network' including the glutamatergic-cholinergic interactions and the prefrontal output neurons that are required for mediation of the performance effects (in the rat cue detection task) are quite disrupted," he wrote in an e-mail to ARF. "To use a simple analogy, it is difficult to enhance the workings of a component of a circuit if the circuit is in a state of advanced disintegration." As for the $\alpha 7$ agonist (ABT-107), the fact it did not improve task performance in the rat studies does not imply these compounds would be ineffective in other cognitive domains, such as memory, he said.

Despite these nuances, Sarter believes his animal setup could be useful in screening for nAChR-stimulating cognition enhancers. "The rats do the detection task. You see the transients. You give the drug. You see what it does to transients and performance at the same time," he said. "You look for drugs that make (transients) bigger and sharper." When it comes to targeting the cholinergic system for therapeutics, it is too simplistic to think in terms of having too much or too little 
neurotransmitter, he said. Compounds should work if they allow proper orchestration of the transients.

Sarter's team has preliminary data suggesting that the transients could have relevance in a disease context. In a rat neurodevelopmental model for schizophrenia that performs poorly in attention tasks, nicotine treatment failed to generate cholinergic transients. Thus far, standard antipsychotics also have been unable to bring back these transients. Any compound that could restore the cholinergic bursts could thus be interesting to pursue, Sarter said.

\section{REFERENCES}

[1] Pigino G, Morfini G, Atagi Y, Deshpande A, Yu C, Jungbauer L, Ladu M, Busciglio J, Brady S (2009) Disruption of fast axonal transport is a pathogenic mechanism for intraneuronal amyloid beta. Proc Natl Acad Sci U S A 106, 5907-5912.

[2] Moreno H, Yu E, Pigino G, Hernandez AI, Kim N, Moreira JE, Sugimori M, Llinás RR (2009) Synaptic transmission block by presynaptic injection of oligomeric amyloid beta. Proc Natl Acad Sci U S A 106, 5901-5906.

[3] Kayed R, Head E, Thompson JL, McIntire TM, Milton SC, Cotman CW, Glabe CG (2003) Common structure of soluble amyloid oligomers implies common mechanism of pathogenesis. Science 300, 486-489.

[4] Kayed R, Pensalfini A, Margol L, Sokolov Y, Sarsoza F, Head E, Hall J, Glabe C (2009) Annular protofibrils are a structurally and functionally distinct type of amyloid oligomer. $J$ Biol Chem 284, 4230-4237.

[5] Santacruz K, Lewis J, Spires T, Paulson J, Kotilinek L, Ingelsson M, Guimaraes A, Deture M, Ramsden M, McGowan E, Forster C, Yue M, Orne J, Janus C, Mariash A, Kuskowski M, Hyman B, Hutton M, Ashe KH (2005) Tau suppression in a neurodegenerative mouse model improves memory function. Science 309, 476-481.

[6] Berger Z, Roder H, Hanna A, Carlson A, Rangachari V, Yue M, Wszolek Z, Ashe K, Knight J, Dickson D, Andorfer C, Rosenberry TL, Lewis J, Hutton M, Janus C (2007) Accumulation of pathological tau species and memory loss in a conditional model of tauopathy. J Neurosci 27, 3650-3662.

[7] Kayed R, Jackson GR (2009) Prefilament tau species as potential targets for immunotherapy for Alzheimer disease and related disorders. Curr Opin Immunol 21, 359-363.

[8] Klucken J, Shin Y, Masliah E, Hyman BT, McLean PJ (2004) Hsp70 reduces alpha-synuclein aggregation and toxicity. $J$ Biol Chem 279, 25497-25502.

[9] Sabri O, Kendziorra K, Wolf H, Gertz HJ, Brust P (2008) Acetylcholine receptors in dementia and mild cognitive impairment. Eur J Nucl Med Mol Imaging 35 Suppl 1, S30-S45.

[10] Wang HY, Stucky A, Liu J, Shen C, Trocme-Thibierge C, Morain P (2009) Dissociating beta-amyloid from alpha 7 nicotinic acetylcholine receptor by a novel therapeutic agent, $\mathrm{S}$ 24795, normalizes alpha 7 nicotinic acetylcholine and NMDA receptor function in Alzheimer's disease brain. J Neurosci 29, 10961-10973.

[11] Dziewczapolski G, Glogowski CM, Masliah E, Heinemann SF (2009) Deletion of the alpha 7 nicotinic acetylcholine receptor gene improves cognitive deficits and synaptic pathology in a mouse model of Alzheimer's disease. J Neurosci 29, 88058815 .

[12] Liu Q, Huang Y, Xue F, Simard A, DeChon J, Li G, Zhang J, Lucero L, Wang M, Sierks M, Hu G, Chang Y, Lukas RJ, Wu J (2009) A novel nicotinic acetylcholine receptor subtype in basal forebrain cholinergic neurons with high sensitivity to amyloid peptides. J Neurosci 29, 918-929.

[13] Bencherif M, Lippiello PM (2010) Alpha7 neuronal nicotinic receptors: The missing link to understanding Alzheimer's etiopathology? Med Hypotheses 74, 281-285.

[14] Parikh V, Kozak R, Martinez V, Sarter M (2007) Prefrontal acetylcholine release controls cue detection on multiple timescales. Neuron 56, 141-154. 